\title{
Oridonin upregulates PTEN through activating p38 MAPK and inhibits proliferation in human colon cancer cells
}

\author{
QIU-XIANG WU ${ }^{1,2}$, SHUANG-XUE YUAN ${ }^{1,2}$, CHUN-MEI REN $^{1,2}$, YU YU ${ }^{1,3}$, \\ WEN-JUAN SUN ${ }^{1,2}$, BAI-CHENG HE ${ }^{1,2}$ and KE WU ${ }^{1,2}$ \\ ${ }^{1}$ Chongqing Municipal Key Laboratory of Higher Education Institutions for Biochemistry and Molecular Pharmacology; \\ Departments of ${ }^{2}$ Pharmacology and ${ }^{3}$ Chemistry, School of Pharmacy, Chongqing Medical University, \\ Yuzhong, Chongqing 400016, P.R. China
}

Received December 10, 2015; Accepted January 12, 2016

DOI: $10.3892 /$ or.2016.4735

\begin{abstract}
Oridonin (ORI) has been reported as an antiproliferation and apoptosis-inducing natural product in various cancer cells. However, the exact molecular mechanism underlying these effects remains unclear. In the present study, we demonstrated the antiproliferation effect of ORI in HCT116 cells, and analyzed the possible molecular mechanism which mediates this effect. We found that ORI inhibits proliferation, induces cell cycle arrest and apoptosis in HCT116 cells, thus also tumor growth. Mechanically, we found that ORI has no substantial effect on mRNA expression of phosphatase and tensin homologue (PTEN), but increases the total protein level of PTEN and markedly reduces the phosphorylation of PTEN; Exogenous expression of PTEN potentiates the anticancer effect of ORI, while knockdown of PTEN attenuates it. ORI also increases the phosphorylation of p38 MAPK, and p38 MAPK-specific inhibitor reduces the antiproliferation effect ORI in HCT116 cells. Moreover, inhibition of p38 MAPK increases the phosphorylation of PTEN, and reverses ORI-induced decrease of PTEN phosphorylation. Our findings suggested that ORI may be a potential anticancer drug for colon cancer, this effect may be mediated by enhancing the function of PTEN through reducing its phosphorylation, which may be resulted from the ORI-induced activation of p38 MAPK.
\end{abstract}

\section{Introduction}

Colon cancer is one of the leading malignancies in the digestive system, and in cancer-related mortality. The current

Correspondence to: $\mathrm{Dr} \mathrm{Ke} \mathrm{Wu}$ or Professor Bai-Cheng $\mathrm{He}$, Department of Pharmacology, School of Pharmacy, Chongqing Medical University, 1 Yixueyuan Road, Yuzhong, Chongqing 400016, P.R. China

E-mail: wukecqmu@163.com

E-mail: hebaicheng99@yahoo.com; 894704897@qq.com

Key words: oridonin, colon cancer, proliferation inhibition, PTEN, p38 MAPK treatment for colon cancer includes surgery, radiotherapy, chemotherapy and targeted therapy alone, or combination. The treatment regimens for the late stage of colon cancer include chemotherapy drugs, such as capecitabine, fluorouracil, irinotecan, oxaliplatin and tegafur (1) or targeted therapy drugs, such as bevacizumab, cetuximab and panitumumab (2). Although the treatment for colon cancer has been developed greatly, the prognosis remains unsatisfactory $(3,4)$. Thus, there is still a great clinical need to develop drugs for colon cancer treatment, particularly for the late stage of colon cancer.

Traditional herb medicine or herbal derived components have played an increasingly important role in the prevention and treatment of cancers, such as camptothecin, vincristine and taxol $(5,6)$. In particular, the combination of these medicines with traditional chemotherapy drugs has substantially improved the prognosis of some cancers. Oridonin (ORI), a diterpenoid extracted from Chinese herb Rabdosia rubescens and/or related species, has attracted increasing attention in the last decades for cancer biologists because of its notable anticancer activity. It has been reported that ORI can induce apoptosis in various cancer cell lines, such as colon cancer cells (7), lymphoma cells (8), breast cancer cells (9) and leukemia cells (10), and this activity may be mediated by inactivating PI3K/Akt and extracellular signal-regulated kinase (ERK), activating p38 mitogen-activated protein kinases (MAPKs), and increasing hydrogen peroxide (11-14). Although substantial evidence supports that ORI can inhibit the proliferation of colon cancer, the precise mechanism underlying this process remains unclear.

In the present study, we investigated the proliferation inhibitory effect of ORI in human colon cells, and dissected the possible mechanism underlying this effect. Our findings confirmed that ORI can inhibit the proliferation of colon cancer cells and demonstrated that this activity may be mediated by upregulating phosphatase and tensin homologue (PTEN) through at least partly activating p38 MAPK.

\section{Materials and methods}

Chemicals and drug preparations. ORI was from Hao-Xuan Bio-Tech Co., Ltd. (Xi'an, China). HCT116 cell line was 
purchased from the American Type Culture Collection. (ATCC; Manassas, VA, USA). ORI was dissolved in dimethyl sulfoxide (DMSO) or prepared with $0.4 \%$ carboxymethylcellulose sodium (CMC-Na) as suspension for in vitro or in vivo experiments, respectively. All antibodies were ordered from Santa Cruz Biotechnology (Santa Cruz, CA, USA). SB203580 (\#S1076) was ordered from Selleckchem (Houston, TX, USA). Cells were maintained in Dulbecco's modified Eagle's medium (DMEM) at $37^{\circ} \mathrm{C}$ in $5 \% \mathrm{CO}_{2}$, with $10 \%$ fetal bovine serum (FBS), $100 \mathrm{U} / \mathrm{ml}$ of penicillin and $100 \mu \mathrm{g} / \mathrm{ml}$ of streptomycin.

Cell viability assay. Cell viability was measured using crystal violet staining. Briefly, HCT116 cells were seeded into a 24-well plate and treated with different concentrations of ORI, the same volume of DMSO was used as solvent control. Cells were washed carefully with cold $\left(4^{\circ} \mathrm{C}\right)$ phosphate-buffered saline (PBS) and stained with $0.5 \%$ crystal violet formalin solution at room temperature to visualize the cell viability at the scheduled time points. For quantification, the crystal violet was extracted with $1 \mathrm{ml} 20 \%$ acetic acid at room temperature for 20 min with shaking. The absorbance at $570 \mathrm{~nm}$ was measured. Each assay was carried out in triplicate.

Construction of recombinant adenovirus. Recombinant adenoviruses expressing PTEN (AdPTEN) and small interference RNA fragments targeting PTEN (AdsiPTEN) were constructed with the AdEasy system (15), tagged with green fluorescence protein (GFP) or red fluorescence protein (RFP), respectively. The recombinant adenovirus expression GFP only was used as vector control.

Flow cytometric analysis for apoptosis and cell cycle. HCT116 cells were seeded in a 6-well plate. For apoptosis assay, cells were treated with different concentrations of ORI or DMSO for $48 \mathrm{~h}$. Then, cells were harvested, washed with cold PBS, and incubated with Annexin V-EGFP and propidium iodide (PI) following the introduction of the kits (KeyGen Biotech, Nanjing, China). The cells were subsequently analyzed by fluorescence activated cell sorting (FACS). For cell cycle analysis, cells were treated with different concentrations of ORI for $24 \mathrm{~h}$. Then, cells were collected, washed with PBS, fixed with cold $\left(4^{\circ} \mathrm{C}\right) 70 \%$ ethanol, washed with 50 and $30 \%$ ethanol, and PBS. Finally, stained with $1 \mathrm{ml}$ of PI $(20 \mathrm{mg} / \mathrm{ml})$ containing RNase $(1 \mathrm{mg} / \mathrm{ml})$ in PBS for $30 \mathrm{~min}$, and followed by FACS for cycle analysis. Each assay was carried out in triplicate.

Western blot assay. Sub-confluent HCT116 cells were seeded in 6-well plate and treated with different concentrations of ORI or DMSO. At the scheduled time points, cells were washed with cold PBS and lysed with $300 \mu 1$ lysis buffer. Cell lysates were boiled for $10 \mathrm{~min}$, then subjected to SDS-PAGE separation and transfered to polyvinylidene fluoride (PVDF) membranes. The membranes were blotted with corresponding primary antibodies, followed by incubating with HRP-conjugated second antibodies. The target proteins were developed with the SuperSignal West Pico Substrate (Pierce, Rockford, IL, USA). Each assay was carried out in triplicate.

Reverse transcription and polymerase chain reaction analysis (RT-PCR). Cells were seeded in T25 flasks and treated with different concentrations of ORI or DMSO. At the corresponding time points, total RNA was extracted with TRIzol reagents (Invitrogen, Carlsbad, CA, USA) and subjected to RT reaction for cDNA templates. Then, the cDNAs were used for detecting the expression level of target genes by PCR. The primer sequences are available upon request. Each assay was carried out in triplicate.

Xenograft tumor model of human colon cancer. The animal experiment was approved by the Institutional Animal Care and Use Committee (IACUC) of Chongqing Medical University. Athymic nude mice (female, 4-6 weeks old) were from the animal centre of Chongqing Medical University (Chongqing, China). Cells were harvested and resuspended in cold PBS to $2 \times 10^{7}$ cells $/ \mathrm{ml}$. Cells in $50 \mu \mathrm{l}$ of cold PBS were injected into the flank of athymic mice (16). One week after injection, animals were treated with different dose of ORI (50 or $100 \mathrm{mg} / \mathrm{kg}$ ) or solvent by intragastric administration, once a day. Four weeks after injection, all animals were sacrificed and the tumors were harvested for histological evaluation.

Histological evaluation and immunohistochemical staining. Retrieved tumor masses were fixed with $10 \%$ formalin and embedded with paraffin. Serial sections were stained with hematoxylin and eosin (H\&E). For immunohistochemical staining, slides were deparaffinized and rehydrated in a graduated fashion, then subjected to antigen retrieval and probed with PTEN antibody or isotype IgG as control. Finally, incubated with biotinylated secondary antibodies and streptavidin conjugated horseradish peroxidase. PTEN was visualized with DAB staining and imaged under a microscope.

Statistical analysis. The results of all experiments are expressed as the mean \pm standard deviation (SD) of at least three independent tests. A Student's t-test was used for single-variable comparisons, and a p-value $<0.05$ was considered statistically significant.

\section{Results}

ORI inhibits the proliferation of HCT116 cells. We explored the antiproliferation effect of ORI on HCT116 cells to validate whether ORI could be used as chemotherapeutic agent for human colon cancer. The results of crystal violet staining showed that ORI effectively inhibits the proliferation of HCT116 cells time- and concentration-dependently (Fig. 1A and B). Cell cycle analysis results showed that ORI induces cell cycle arrest apparently at S phase in HCT116 cells (Fig. 1C). Western blot assay showed that ORI also significantly suppresses the expression of proliferating cell nuclear antigen (PCNA) (Fig. 1D). These data suggested ORI is capable of inhibiting proliferation in HCT116 cells.

ORI induces apoptosis in HCT116 cells. We next tested whether ORI could induce HCT116 cells to undergo apoptosis. HCT116 cells were treated with different concentrations of ORI or DMSO for 24 or $48 \mathrm{~h}$. Then, cells were subjected with flow cytometry or western blot analyses. The results showed that ORI increases the apoptotic cell rate concentration- dependently (Fig. 2A). Western blot analysis 

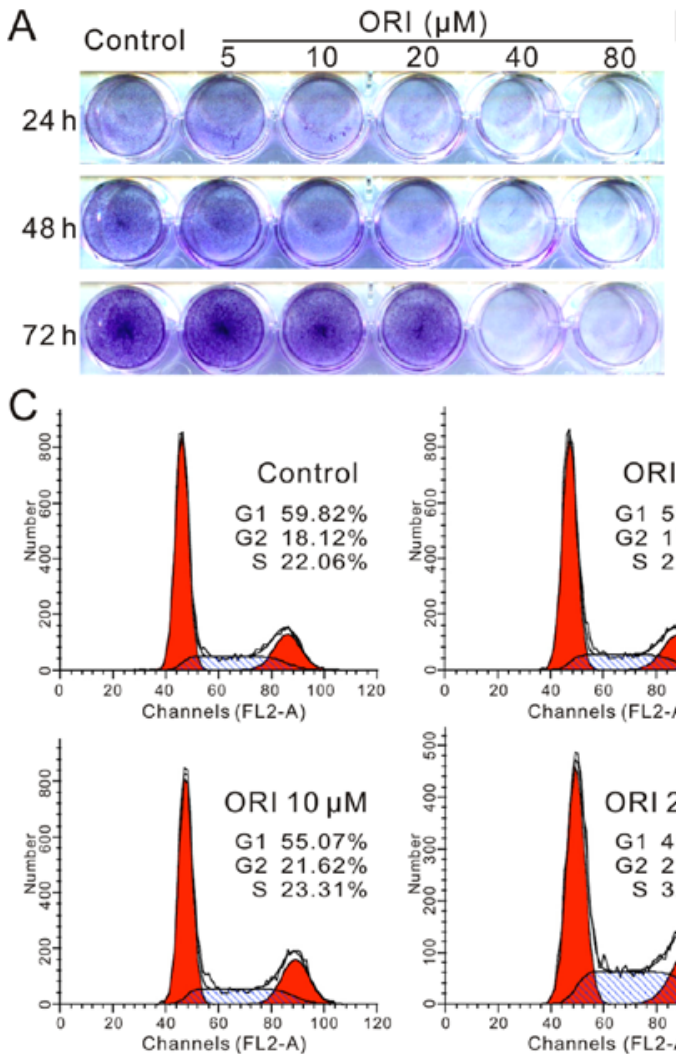

B

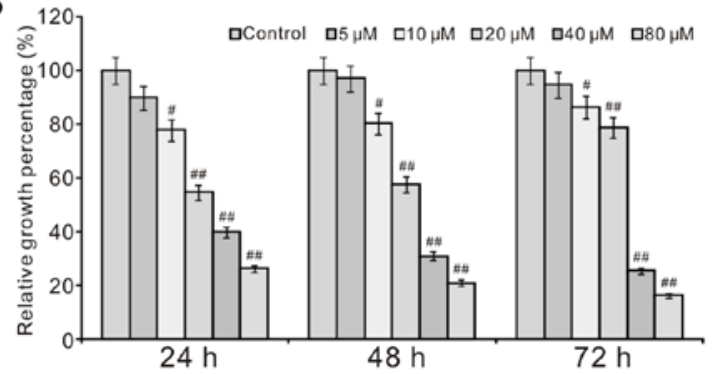

Figure 1. Effects of ORI on the proliferation in HCT116 cells. (A) Crystal violet staining results show the antiproliferation effect of ORI in HCT116 cells (B) Quantitative results of crystal violet staining show the antiproliferation effect of ORI in HCT116 cells ( ${ }^{\#} \mathrm{p}<0.05$, compared with control; ${ }^{\# \#} \mathrm{p}<0.01$, compared with control). (C) Flow cytometric analysis shows the cell cycle arrest effect of ORI in HCT116 cells. (D) Western blot assay shows the effect of ORI on PCNA level in HCT116 cells. GAPDH was used as loading control. The assays were performed in triplicate.

A
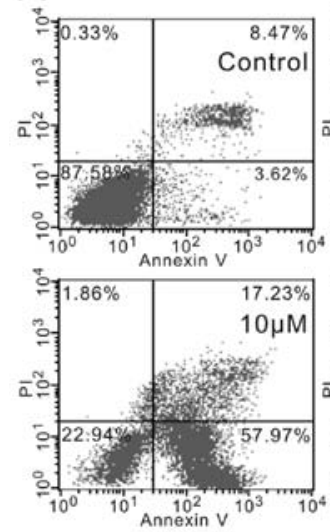
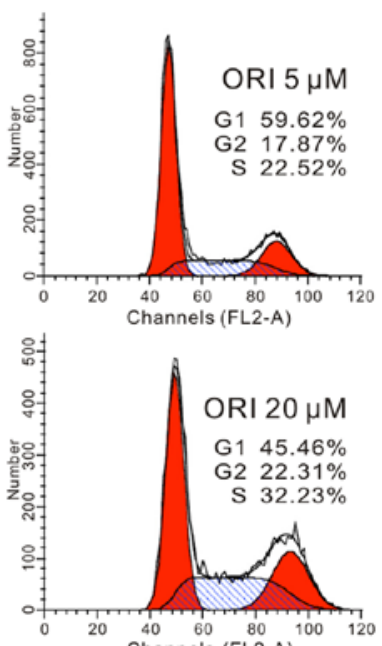

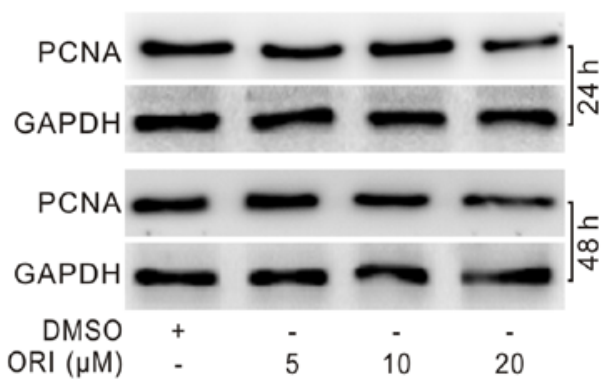

Figure 2. Effects of ORI on apoptosis in HCT116 cells. (A) Flow cytometric analysis shows the apoptosis induced by ORI in HCT116 cells. (B) Western blot assay shows the protein level of Bad and Bcl-2 affected by ORI in HCT116 cells. GAPDH was used as loading control. The assays were performed in triplicate.

showed that ORI substantially increases the level of Bad and reduces the level of Bcl-2 in HCT116 cells (Fig. 2B). These results indicated that ORI is a potent apoptosis inducer for colon cancer cells.

ORI inhibits tumor growth in an ectopic human cancer model. We next investigated the in vivo anticancer activity of ORI with a well-established xenograft colon cancer model (17). We injected HCT116 cells subcutaneously into the flanks of athymic nude mice. One week after injection, the mice
B

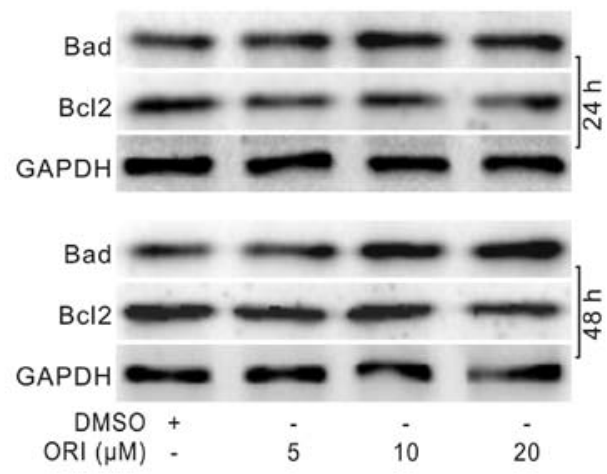

were treated with different doses of ORI (50 or $100 \mathrm{mg} / \mathrm{kg}$ ) through intragastric administration, once a day up to 4 weeks. Suspension of $0.4 \%$ CMC-Na were administrated as control. The results showed that tumor masses of ORI treated group are smaller than those of the control group, and ORI inhibits the tumor growth dose dependently (Fig. 3A). H\&E staining results showed that more necrotic cells were found in ORI treated group, comparing with the solvent group (Fig. 3B). These in vivo results further demonstrated that ORI may be a potential anticancer reagent for colon cancer treatment. 


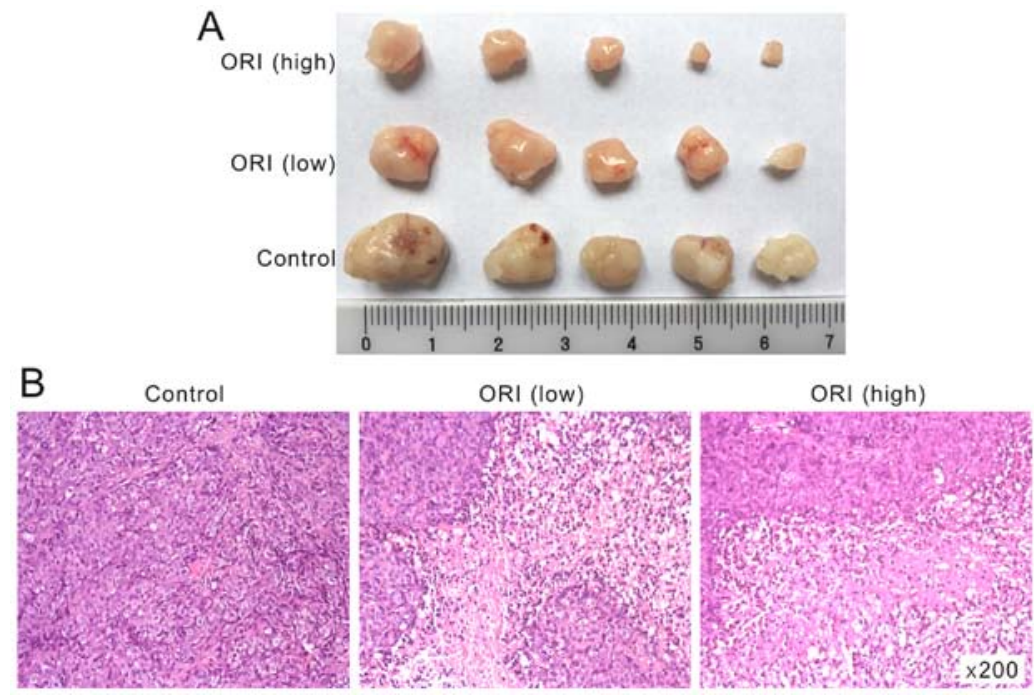

Figure 3. Effects of ORI on tumor growth of colon cancer. (A) Tumor masses retrieved from nude mice show the anticancer effect of ORI on colon cancer. (B) H\&E staining results show the anticancer effect of ORI on colon cancer. Representative results are shown.
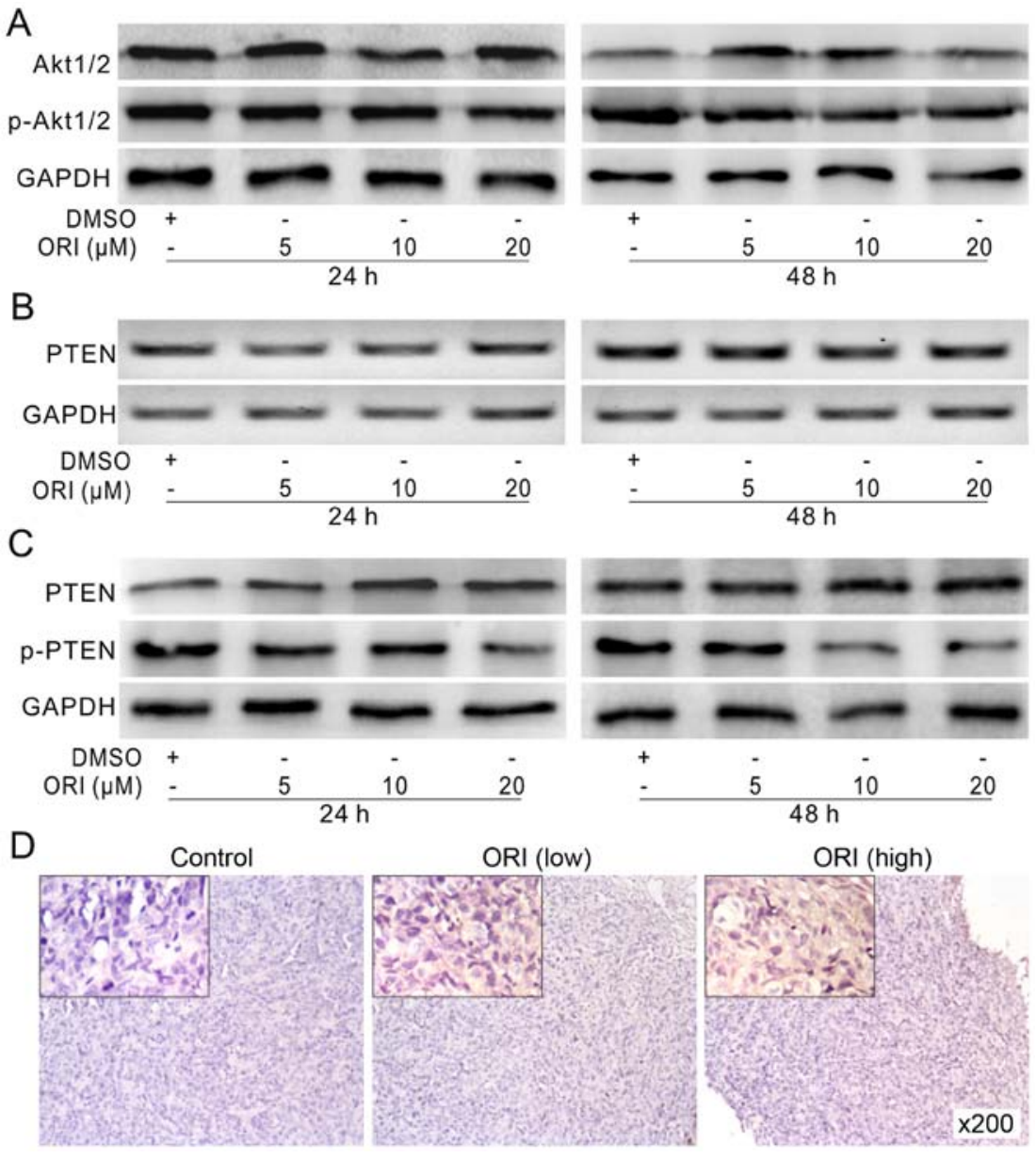

Figure 4. Effects of ORI on the level of PTEN in HCT116 cells. (A) Western blot assay shows the effect of ORI on Akt1/2 and p-Akt1/2 in HCT116 cells. GAPDH was used as loading control. (B) PCR analysis results show the effect of ORI on the expression of PTEN in HCT116 cells. GAPDH was used as loading control. (C) Western blot results show the effect of ORI on the level of PTEN and p-PTEN in HCT116 cells. GAPDH was used as loading control. (D) Immunohistochemical staining results show the effect of ORI on PTEN in colon cancer, representative results are shown. All assay were performed in triplicate.

ORI upregulates PTEN in HCT116 cells. Cell proliferation is well-regulated by various signalings or growth factors.
Using western blot assay, we found that ORI can reduce the phosphorylation of Akt1/2 (Fig. 4A), which suggested that the 


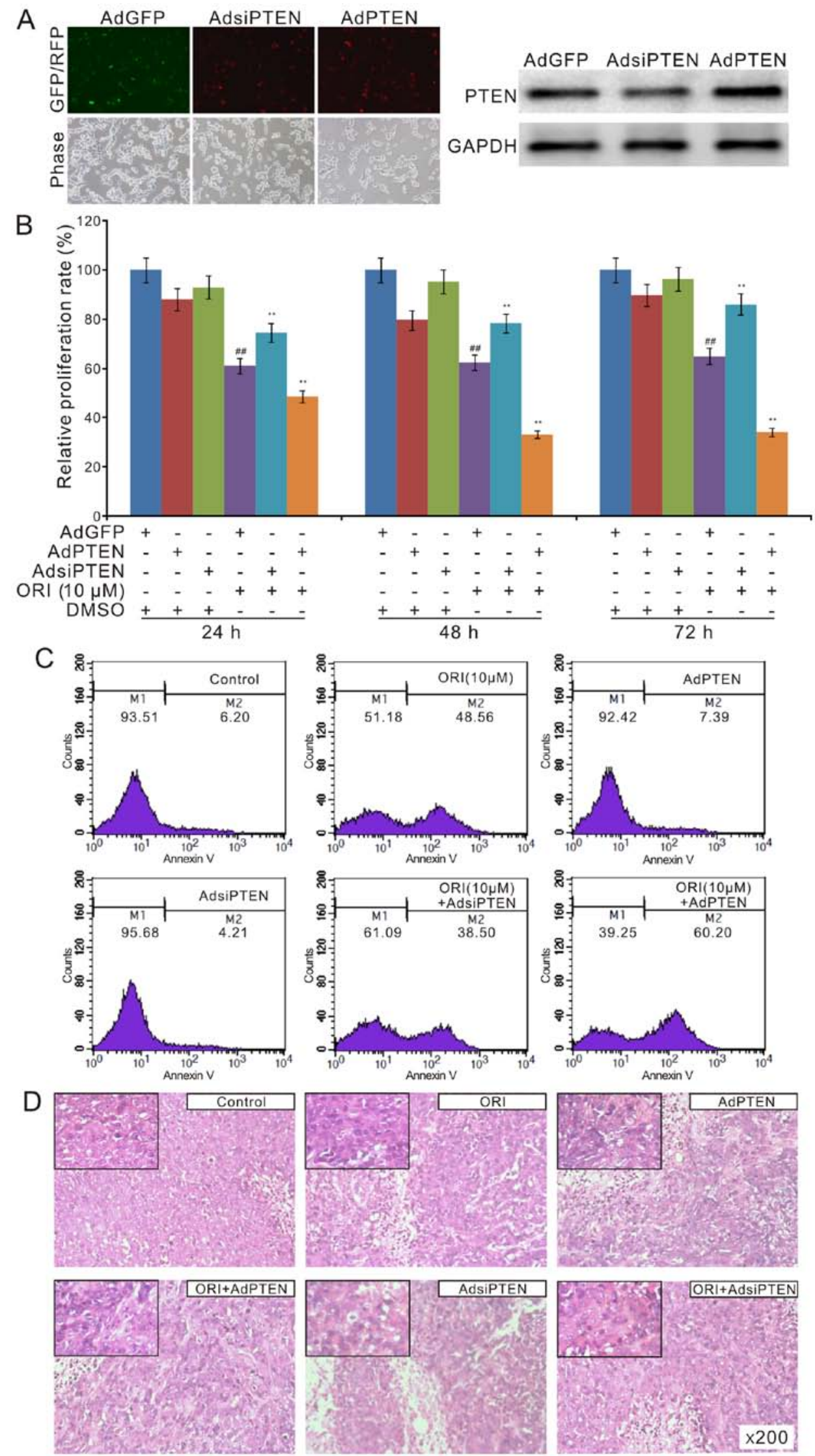

Figure 5. Effects of PTEN on the anticancer activity of ORI in colon cancer. (A) Fluorescence images and western blot analysis shows the effect of recombinant adenoviruses on the expression of PTEN in HCT116 cells. Left panel shows the infection results of recombinant adenovirus in HCT116 cells, right panel show the effect of the adenoviruses on the expression of PTEN in HCT116 cells. (B) Quantification results of crystal violet staining show the antiproliferation effect of ORI affected by exogenous expression of PTEN or PTEN knockdown in HCT116 cells $\left({ }^{\# \#} \mathrm{p}<0.01\right.$, compared with control; ${ }^{* *} \mathrm{p}<0.01$, compared with ORI treated group). (C) Flow cytometric analysis shows the apoptosis-inducing effect of ORI affected by exogenous expression of PTEN or PTEN knockdown in HCT116 cells. (D) H\&E staining results show the anticancer effect of ORI affected by exogenous expression of PTEN or PTEN knockdown in colon cancer. Representative results are shown. 

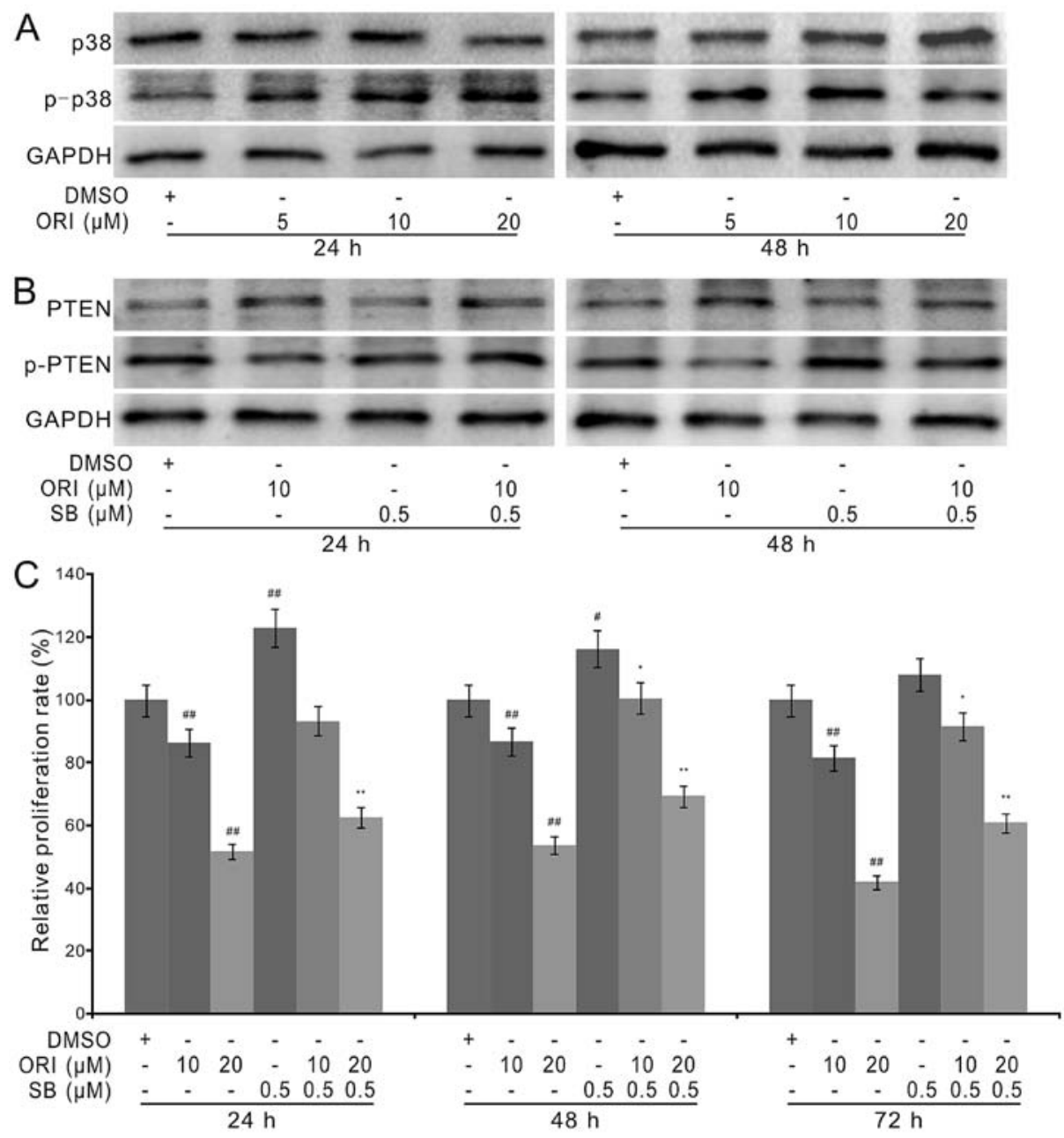

Figure 6. Effects of p38 MAPK on PTEN phosphorylation in HCT116 cells. (A) Western blot results show the effect of ORI on p38 and p-p38 in HCT116 cells. GAPDH was used as loading control. (B) Western blot results show the effect of ORI on p-PTEN and PTEN affected by p38 MAPK inhibitor (SB, p38 MAPK inhibitor, SB203580). GAPDH was used as loading control. (C) Quantification results of crystal violet staining show the antiproliferation effect of ORI affected by 338 MAPK inhibitor in HCT116 cells (SB, p38 MAPK inhibitor, SB203580). The assays were performed in triplicate.

PI3K/Akt signaling was inhibited by ORI in HCT116 cells. PTEN, as a tumor suppressor, can negatively regulate PI3K/ Akt signaling. For this reason, we speculated that the inhibition of PI3K/Akt signaling may be contributed to PTEN upregulation by ORI in HCT116 cells. However, with PCR assay, we found ORI exerts no substantial effect on the mRNA level of PTEN in HCT116 cells (Fig. 4B). Further results showed that ORI can markedly increase the protein level of PTEN and decrease the phosphorylation of PTEN, concentrationand time-dependently (Fig. 4C). The immunohistochemical staining results showed that PTEN is upregulated also in colon cancer dose-dependently (Fig. 4D). These results suggested that the antiproliferation effect of ORI in colon cancer cells may be associated with PTEN upregulation.

PTEN partly mediates the antiproliferation effect of ORI in HCT116 cells. We next investigated the role of PTEN in the antiproliferation effect ORI in colon cancer cells. We constructed recombinant adenoviruses of PTEN and siRNA fragments for PTEN. Western blot results showed that all the recombinant adenoviruses exert their function well (Fig. 5A). The crystal violet staining analysis showed that exogenous expression of PTEN substantially enhances the antiprolifera- tion of ORI, while knockdown of PTEN reduces this effect of ORI in HCT116 cells (Fig. 5B). The flow cytometry assay results showed that exogenous expression of PTEN increases the apoptotic cell rate induced by ORI in HCT116 cells. On the contrary, PTEN knockdown reduces ORI-induced apoptosis (Fig. 5C). The H\&E staining results showed that the anticancer effect of ORI was enhanced by exogenous expression of PTEN, but decreased by PTEN knockdown (Fig. 5D). These data suggested that the anticancer effect of ORI may be partly mediated by PTEN upregulation.

ORI upregulates PTEN by activating p38 MAPK in HCT116 cells. Although PTEN can partly mediate the antiproliferation of ORI in HCT116, the mechanism of how ORI upregulates PTEN remains unknown. With further analysis, we found that ORI increases the phosphorylation of p38 MAPK, concentration-dependently (Fig. 6A), increases the level of PTEN, but decreases the phosphorylation of PTEN; p38 MAPK inhibitor shows no substantial effect on the level of PTEN, but obviously increases its phosphorylation. When ORI was combined with $\mathrm{p} 38$ MAPK inhibitor, the level of p-PTEN was clearly increased, although no substantial effect on the level of PTEN was observed (Fig. 6B). Crystal violet staining analysis 
results showed that p38 MAPK inhibitor partly reverses the antiproliferation effect of ORI in HCT116 cells (Fig. 6C), which is similar with the effect of PTEN knockdown on the antiproliferation effect of ORI (Fig. 5B). These data suggested that ORI-induced upregulation of PTEN may be resulted from activating p38 MAPK, which may block the phosphorylation of PTEN in colon cancer cells.

\section{Discussion}

In the present study, we demonstrated that ORI can be used as a potential antiproliferation drug for colon cancer cells. Mechanistically, we found that the anticancer effect of ORI may be partly mediated by upregulating PTEN through activating p38 MAPK to reduce PTEN phosphorylation.

ORI has been used as a drug for antitumor, anti-microbial, anti-inflammatory and antioxidant for many years (18). It has been reported that ORI can inhibit proliferation and induce apoptosis in various cancer cells, such as breast, pancreatic, lung, gastric and prostate cancer $(8,19-23)$, as well as induce apoptosis and senescence in colon cancer cells $(12,24)$. Our data also confirmed that ORI can inhibit proliferation and induce apoptosis in HCT116 cells concentration-dependently, as well as inhibit the tumor growth of colon cancer. Therefore, ORI may be a promising natural product as chemotherapy drug for colon cancer treatment. As reported, ORI is a multitarget natural product (18), and the anticancer effect of ORI may be mediated by upregulating p53 and p21, decreasing Bcl-2 and epidermal growth factor receptor, inhibiting Wnt/ $\beta$-catenin and PI3K/Akt signaling (25-29). Although the anticancer activity of ORI in colon cancer may also be associated with upregulating p21 and inhibiting Wnt/ $\beta$-catenin signaling $(12,24)$, the precise mechanism underlying this process remains unclear.

$\mathrm{PI} 3 \mathrm{~K} / \mathrm{Akt}$ signal is an essential pathway regulating proliferation, apoptosis and survival. It has been targeted for many anticancer drugs (29-31). PI3K/Akt signaling can be negatively regulated by PTEN, another important tumor suppressor. The mutation and/or function loss of PTEN will lead to PI3K/Akt signaling over-activation and uncontrolled proliferation. Hence, PTEN is an important target for cancer control, and some promising chemotherapy drugs have been used to target PTEN (32). In the present study, we found that ORI can reduce $\mathrm{p}-\mathrm{Akt} 1 / 2$ (Fig. 4A), which suggested that the anticancer activity of ORI in colon cancer cells may result from inactivating PI3K/Akt signaling. Although PTEN is one of the major negative regulators for PI3K/Akt signaling, we found ORI exerts no significant change on the mRNA level of PTEN. Further analysis indicated that ORI can substantially increase the protein level of PTEN and reduce the level of p-PTEN. Thus, our data indicated that the anticancer activity of ORI in colon cancer may be mediated by inactivating PI3K/Akt signaling through upregulating PTEN, which may be the result of decreasing the phosphorylation of PTEN.

PTEN acts as a lipid phosphatase to catalyse PIP3 dephosphorylation resulting in the production of PIP2. One of its main physiological function is to negatively regulate PI3K/Akt signaling (33). For colon cancer, the reduction or mutation of PTEN plays an essential role at the early stage of sporadic colorectal carcinogenesis (34), and the highly expressed
PTEN is also associated with the chemosensitivity of colon cancer (35). Therefore, PTEN may be a potential therapeutical target for colon cancer treatment. PTEN is well regulated by various mechanisms, such as transcriptional regulation and post-transcriptional regulation, and phosphorylation (36). However, the detail of each regulation process still need to be fully unveiled. Our results showed that ORI may increase the level of PTEN by reducing its phosphorylation (Fig. 4C). p38 MAPK is a critical mediator for signaling transduction, which responds to a wide range of extracellular stresses such as UV radiation, hypoxia and oxidative stress (37). It has been reported that the activation of p38 MAPK mediates the anticancer activity of ORI in breast cancer (38). Our previous study indicated that bone morphogenetic protein 9 (BMP9), the most potent osteogenic protein, can activate p38 MAPK and downregulates PTEN in mesenchymal stem cells (39). However, the relationship between p38 MAPK and PTEN in cancer remains unknown. Thus, we hypothesized that p38 MAPK may interact with PTEN directly or indirectly in cancer. Using western blot assay, we found that ORI can activate p38 MAPK in HCT116 cells, as well as upregulate PTEN (Fig. 6A). With further analysis, we found that ORI can reduce the level of p-PTEN, which can be partly reversed by p38 MAPK inhibitor (Fig. 6B). Inhibition of p38 MAPK can partly reverse the antiproliferation effect of ORI in HCT116 cells (Fig. 6C). These results indicated that upregulation of PTEN and activation of p38 MAPK are both involved in the antiproliferation effect of ORI in HCT116 cells. Therefore, our data strongly suggested that ORI-induced upregulation of PTEN may be the result of reducing the phosphorylation of PTEN, which may be mediated by the ORI-induced activation of p38 MAPK.

In summary, we demonstrated that ORI can be used as an effective chemotherapy drug for human colon cancer. The anticancer activity of ORI in colon cancer may be mediated by increasing the level of PTEN, which may result from the ORI-induced activation of p38 MAPK. Future studies should be carried out to investigate the possible molecular mechanism of ORI on the activation of p38 MAPK, and decipher the possible interaction between p38 MAPK and PTEN phosphorylation in colon cancer cells.

\section{Acknowledgements}

We thank Professor Tong-Chuan He of the University of Chicago Medical Center (Chicago, IL, USA) for his kind provision of the recombinant adenoviruses. The present study was supported by the Research Grant from the National Natural Science Foundation of China (grant nos. NSFC 81372120 and 81572226 to Bai-Cheng He).

\section{References}

1. Chemotherapy of metastatic colorectal cancer. Prescrire Int 19: 219-224, 2010.

2. Tran NH, Cavalcante LL, Lubner SJ, Mulkerin DL, LoConte NK, Clipson L, Matkowskyj KA and Deming DA: Precision medicine in colorectal cancer: The molecular profile alters treatment strategies. Ther Adv Med Oncol 7: 252-262, 2015.

3. Binefa G, Rodríguez-Moranta F, Teule A and Medina-Hayas M: Colorectal cancer: From prevention to personalized medicine. World J Gastroenterol 20: 6786-6808, 2014. 
4. Feng QY, Wei Y, Chen JW, Chang WJ, Ye LC, Zhu DX and $\mathrm{Xu}$ JM: Anti-EGFR and anti-VEGF agents: Important targeted therapies of colorectal liver metastases. World J Gastroenterol 20: 4263-4275, 2014.

5. Banjerdpongchai R, Chanwikruy Y, Rattanapanone V and Sripanidkulchai B: Induction of apoptosis in the human Leukemic U937 cell line by Kaempferia parviflora Wall.ex.Baker extract and effects of paclitaxel and camptothecin. Asian Pac J Cancer Prev 10: 1137-1140, 2009.

6. Weaver BA: How Taxol/paclitaxel kills cancer cells. Mol Biol Cell 25: 2677-2681, 2014.

7. Zhu Y, Xie L, Chen G, Wang H and Zhang R: Effects of oridonin on proliferation of HT29 human colon carcinoma cell lines both in vitro and in vivo in mice. Pharmazie 62: 439-444, 2007.

8. Liu YQ, Mu ZQ, You S, Tashiro S, Onodera S and Ikejima T: Fas/FasL signaling allows extracelluar-signal regulated kinase to regulate cytochrome $c$ release in oridonin-induced apoptotic U937 cells. Biol Pharm Bull 29: 1873-1879, 2006.

9. Hsieh TC, Wijeratne EK, Liang JY, Gunatilaka AL and Wu JM Differential control of growth, cell cycle progression, and expression of NF-kappaB in human breast cancer cells MCF-7, MCF-10A, and MDA-MB-231 by ponicidin and oridonin, diterpenoids from the Chinese herb Rabdosia rubescens. Biochem Biophys Res Commun 337: 224-231, 2005.

10. Zhou GB, Kang H, Wang L, Gao L, Liu P, Xie J, Zhang FX, Weng XQ, Shen ZX, Chen J, et al: Oridonin, a diterpenoid extracted from medicinal herbs, targets AML1-ETO fusion protein and shows potent antitumor activity with low adverse effects on $\mathrm{t}(8 ; 21)$ leukemia in vitro and in vivo. Blood 109 : 3441-3450, 2007.

11. Hu HZ, Yang YB, Xu XD, Shen HW, Shu YM, Ren Z, Li XM, Shen HM and Zeng HT: Oridonin induces apoptosis via PI3K/Akt pathway in cervical carcinoma HeLa cell line. Acta Pharmacol Sin 28: 1819-1826, 2007.

12. Gao FH, Liu F, Wei W, Liu LB, Xu MH, Guo ZY, Li W, Jiang B and $\mathrm{Wu}$ YL: Oridonin induces apoptosis and senescence by increasing hydrogen peroxide and glutathione depletion in colorectal cancer cells. Int J Mol Med 29: 649-655, 2012.

13. Cheng Y, Qiu F, Ye YC, Tashiro S, Onodera S and Ikejima T: Oridonin induces $\mathrm{G}_{2} / \mathrm{M}$ arrest and apoptosis via activating ERK-p53 apoptotic pathway and inhibiting PTK-Ras-Raf-JNK survival pathway in murine fibrosarcoma L929 cells. Arch Biochem Biophys 490: 70-75, 2009.

14. Bu HQ, Liu DL, Wei WT, Chen L, Huang H, Li Y and Cui JH: Oridonin induces apoptosis in SW1990 pancreatic cancer cells via p53- and caspase-dependent induction of p38 MAPK. Oncol Rep 31: 975-982, 2014.

15. Luo J, Deng ZL, Luo X, Tang N, Song WX, Chen J, Sharff KA, Luu HH, Haydon RC, Kinzler KW, et al: A protocol for rapid generation of recombinant adenoviruses using the AdEasy system. Nat Protoc 2: 1236-1247, 2007.

16. Wu K, Zhou M, Wu QX, Yuan SX, Wang DX, Jin JL, Huang J, Yang JQ, Sun WJ, Wan LH, et al: The role of IGFBP-5 in mediating the anti-proliferation effect of tetrandrine in human colon cancer cells. Int J Oncol 46: 1205-1213, 2015.

17. He BC, Gao JL, Zhang BQ, Luo Q, Shi Q, Kim SH, Huang E, Gao Y, Yang K, Wagner ER, et al: Tetrandrine inhibits Wnt/ $\beta$ catenin signaling and suppresses tumor growth of human colorectal cancer. Mol Pharmacol 79: 211-219, 2011.

18. Owona BA and Schluesener HJ: Molecular insight in the multifunctional effects of oridonin. Drugs RD 15: 233-244, 2015.

19. Wang S, Zhong Z, Wan J, Tan W, Wu G, Chen M and Wang Y: Oridonin induces apoptosis, inhibits migration and invasion on highly-metastatic human breast cancer cells. Am J Chin Med 41: 177-196, 2013.

20. Liu DL, Bu HQ, Jin HM, Zhao JF, Li Y and Huang H: Enhancement of the effects of gemcitabine against pancreatic cancer by oridonin via the mitochondrial caspase-dependent signaling pathway. Mol Med Rep 10: 3027-3034, 2014.

21. Liu Y, Liu JH, Chai K, Tashiro S, Onodera S and Ikejima T: Inhibition of c-Met promoted apoptosis, autophagy and loss of the mitochondrial transmembrane potential in oridonin-induced A549 lung cancer cells. J Pharm Pharmacol 65: 1622-1642, 2013.
22. Gao SY, Li J, Qu XY, Zhu N and Ji YB: Downregulation of Cdk1 and cyclinB1 expression contributes to oridonin-induced cell cycle arrest at G2/M phase and growth inhibition in SGC-7901 gastric cancer cells. Asian Pac J Cancer Prev 15: 6437-6441, 2014.

23. Li X, Li X, Wang J, Ye Z and Li JC: Oridonin up-regulates expression of $P 21$ and induces autophagy and apoptosis in human prostate cancer cells. Int J Biol Sci 8: 901-912, 2012.

24. Gao FH, Hu XH, Li W, Liu H, Zhang YJ, Guo ZY, Xu MH, Wang ST, Jiang B, Liu F, et al: Oridonin induces apoptosis and senescence in colorectal cancer cells by increasing histone hyperacetylation and regulation of p16, p21, p27 and c-myc. BMC Cancer 10: 610, 2010.

25. Cui Q, Tashiro S, Onodera S, Minami M and Ikejima T: Oridonin induced autophagy in human cervical carcinoma HeLa cells through Ras, JNK, and P38 regulation. J Pharmacol Sci 105: 317-325, 2007.

26. Feng FF, Zhang DR, Tian KL, Lou HY, Qi XL, Wang YC, Duan CX, Jia LJ, Wang FH, Liu Y, et al: Growth inhibition and induction of apoptosis in MCF-7 breast cancer cells by oridonin nanosuspension. Drug Deliv 18: 265-271, 2011

27. Li D, Wu LJ, Tashiro S, Onodera S and Ikejima T: Oridonin induces human epidermoid carcinoma A431 cell apoptosis through tyrosine kinase and mitochondrial pathway. J Asian Nat Prod Res 10: 77-87, 2008.

28. Huang HL, Weng HY, Wang LQ, Yu CH, Huang QJ, Zhao PP, Wen JZ, Zhou H and Qu LH: Triggering Fbw7-mediated proteasomal degradation of c-Myc by oridonin induces cell growth inhibition and apoptosis. Mol Cancer Ther 11: 1155$1165,2012$.

29. Fresno Vara JA, Casado E, de Castro J, Cejas P, Belda-Iniesta C and González-Barón M: PI3K/Akt signalling pathway and cancer. Cancer Treat Rev 30: 193-204, 2004.

30. Osaki M, Oshimura M and Ito H: PI3K-Akt pathway: Its functions and alterations in human cancer. Apoptosis 9: 667-676, 2004

31. Polivka J Jr and Janku F: Molecular targets for cancer therapy in the PI3K/AKT/mTOR pathway. Pharmacol Ther 142: 164-175, 2014.

32. Carnero A, Blanco-Aparicio C, Renner O, Link W and Leal JF: The PTEN/PI3K/AKT signalling pathway in cancer, therapeutic implications. Curr Cancer Drug Targets 8: 187-198, 2008.

33. Milella M,Falcone I, Conciatori F, Cesta Incani U,Del Curatolo A, Inzerilli N, Nuzzo CM, Vaccaro V, Vari S, Cognetti F, et al: PTEN: Multiple functions in human malignant tumors. Front Oncol 5: 24, 2015.

34. Waniczek D, Śnietura M, Młynarczyk-Liszka J, Pigłowski W, Kopeć A, Lange D, Rudzki M and Arendt J: PTEN expression profiles in colorectal adenocarcinoma and its precancerous lesions. Pol J Pathol 64: 15-20, 2013.

35. Hsu CP, Kao TY, Chang WL, Nieh S, Wang HL and Chung YC: Clinical significance of tumor suppressor PTEN in colorectal carcinoma. Eur J Surg Oncol 37: 140-147, 2011.

36. Song MS, Salmena L and Pandolfi PP: The functions and regulation of the PTEN tumour suppressor. Nat Rev Mol Cell Biol 13: 283-296, 2012

37. Tormos AM, Taléns-Visconti R, Nebreda AR and Sastre J: p38 MAPK: A dual role in hepatocyte proliferation through reactive oxygen species. Free Radic Res 47: 905-916, 2013.

38. Cui Q, Tashiro S, Onodera S, Minami M and Ikejima T: Autophagy preceded apoptosis in oridonin-treated human breast cancer MCF-7 cells. Biol Pharm Bull 30: 859-864, 2007.

39. Huang J, Yuan SX, Wang DX, Wu QX, Wang X, Pi CJ, Zou X, Chen L, Ying LJ, Wu K, et al: The role of COX-2 in mediating the effect of PTEN on BMP9 induced osteogenic differentiation in mouse embryonic fibroblasts. Biomaterials 35: 9649-9659, 2014. 